\title{
Soft-Output Successive Cancellation Stack Polar Decoder
}

\author{
Luping Xiang, Member, IEEE, Yusha Liu, Robert G. Maunder, Senior Member, IEEE, Lie-Liang Yang, Fellow, \\ IEEE and Lajos Hanzo, Fellow, IEEE
}

\begin{abstract}
Polar coding has been ratified for employment in the 3GPP New Radio standard and several soft-decision decoders achieved comparable performance to that of the state-of-the-art successive cancellation list decoder. Aiming for further improving the performance of the soft-decision polar decoders, we propose a soft-output successive cancellation stack (SSCS) polar decoder, which jointly exploits the benefits of the depth-first search of the stack decoder and the soft information output of the belief propagation decoder. This has the substantial benefit of facilitating soft-input soft-output (SISO) decoding and seamless iterative information exchange in turbo-style receivers. As a further contribution, we intrinsically amalgamate our SSCS decoder into polarcoded large-scale multiple-input multiple-output (MIMO) systems and conceive an iterative turbo receiver, operating on the basis of logarithmic likelihood ratios (LLRs). Our simulation results show that the proposed SSCS decoder is capable of outperforming the state-of-the-art SISO polar decoders, despite requiring a lower complexity at moderate to high signal-to-noise ratios (SNRs). Additionally, compared with the non-iterative hard-output SCS decoder, our SSCS scheme attained 1.5 dB SNR gain at a bit error ratio level of $10^{-5}$, when decoding the $[256,512]$ polar code of a $(64 \times 64)$ MIMO system.
\end{abstract}

Index Terms-Polar code, successive cancellation stack decoding, soft-input soft-output,multiple-input multiple-output.

\section{INTRODUCTION}

Polar codes [1] have been standardized as one of the channel coding schemes ratified for the 5G uplink control channels [2], hence attracting extensive research interests in high-performance yet low-complexity decoding algorithms [3]. Owing to its intrinsic error propagation deficiency, the original successive cancellation (SC) decoding algorithm [1] suffers from severe bit error ratio (BER) erosion for short block lengths, when compared with low-density parity check (LDPC) or turbo codes [4]. For mitigating this performance degradation, the SC list (SCL) [5] and the SC stack (SCS) [6] polar decoders were conceived by adopting the breadth-first and depth-first search techniques, respectively. Furthermore, sphere decoding based polar decoders have been proposed for attaining near maximum-likelihood performance at a low decoding complexity $[7,8]$.

In order to facilitate the employment of polar decoders in practical communication systems relying on logarithmic domain, processing the logarithmic likelihood ratio (LLR)-based SCL or SCS decoding of polar codes has been investigated in [9-12], which generate hard bit decisions based on their LLR inputs. For facilitating iterative detection and decoding (IDD), soft-output decoders have also been proposed, such as the belief propagation (BP) [13] and soft cancellation (SCAN)

The authors are with School of Electronics and Computer Science, University of Southampton, SO17 1BJ, UK. (E-mail: $1 x 1 \mathrm{~g} 15$, yl6g15, rm, 1ly, lh@soton.ac.uk). (Corresponding author: Yusha Liu)

L. Hanzo would like to gratefully acknowledge the financial support of the Engineering and Physical Sciences Research Council projects EP/Noo4558/1, EP/P034284/1, COALESCE, of the Royal Society's Global Challenges Research Fund Grant as well as of the European Research Council's Advanced Fellow Grant QuantCom (Grant No. 789028).
TABLE I

CONTRASTING OUR CONTRIBUTIONS TO THE STATE-OF-THE ART.

\begin{tabular}{l|c|c|c|c|c}
\hline Contributions & this work & {$[10-12]$} & {$[14]$} & {$[18]$} & {$[19]$} \\
\hline \hline Soft-output & $\checkmark$ & & $\checkmark$ & $\checkmark$ & $\checkmark$ \\
\hline SCS decoder & $\checkmark$ & $\checkmark$ & & & \\
\hline Large-scale MIMO & $\checkmark$ & & & & \\
\hline Reduced complexity & $\checkmark$ & & & & \\
\hline IDD & $\checkmark$ & & $\checkmark$ & & $\checkmark$ \\
\hline
\end{tabular}

[14] polar decoders, which outperformed the non-iterative hardoutput SCL decoder for transmission over fading channels by exploiting extrinsic information exchange between the detector and decoder. By applying early-stopping approaches [15], the complexity of the BP decoding can be further reduced.

More sophisticated soft-output polar decoders have been investigated [16-20]. By designing permuted polar code factor graphs for generating a list of codewords and performing BP decoding, a belief propagation list (BPL) decoder is proposed in [16], which achieves comparable BER performance to that of the SCL decoder. Similarly, by applying SCAN decoding independently to a list of permuted polar codewords, the SCAN list (SCANL) decoding scheme of [17] attains slightly better BER performance than the BPL decoder. Recently, soft-output of the SCL decoder has been achieved in $[18,19]$. Furthermore, Feng et al. [20] proposed an iterative adjustable soft list (IASCL) polar decoder, which relied on the soft cancellation (SCAN) polar decoding principle for generating soft decisions for the entire candidate lists. IDD operating on the basis of LLRs for polar-coded multiple access systems have been studied in [2123].

However, to the best of our knowledge, there exists no open literature on how to generate the soft-output of SCS decoders, even though this would have the potential of reducing the detection complexity of the SCL decoder $[10,11]$. Motivated by filling this knowledge gap in the open literature, we propose a soft-output SCS (SSCS) decoder, which facilitates the iterative decoding of polar-coded systems. In summary, the novel contributions of this compact Letter are boldly and explicitly contrasted to the literature in Table I and are summarized as follows.

- Firstly, we propose a SSCS decoding algorithm for polar codes by concatenating the classic BP scheduling to the SCS decoder. In this way, extrinsic LLRs can be generated during the SCS decoding process which are fed back to the signal detector for IDD operations.

- Furthermore, we intrinsically amalgamate the proposed SSCS polar decoder with a large-scale multiple-input multiple-output (MIMO) vertical-Bell Laboratories layered space-time (V-BLAST) system and conceive an iterative receiver, facilitating the soft extrinsic information exchange within a turbo-style receiver. This is in contrast to the polar-coded MIMO system proposed in [24], which relies on the hard decision outputs of the polar decoder. 


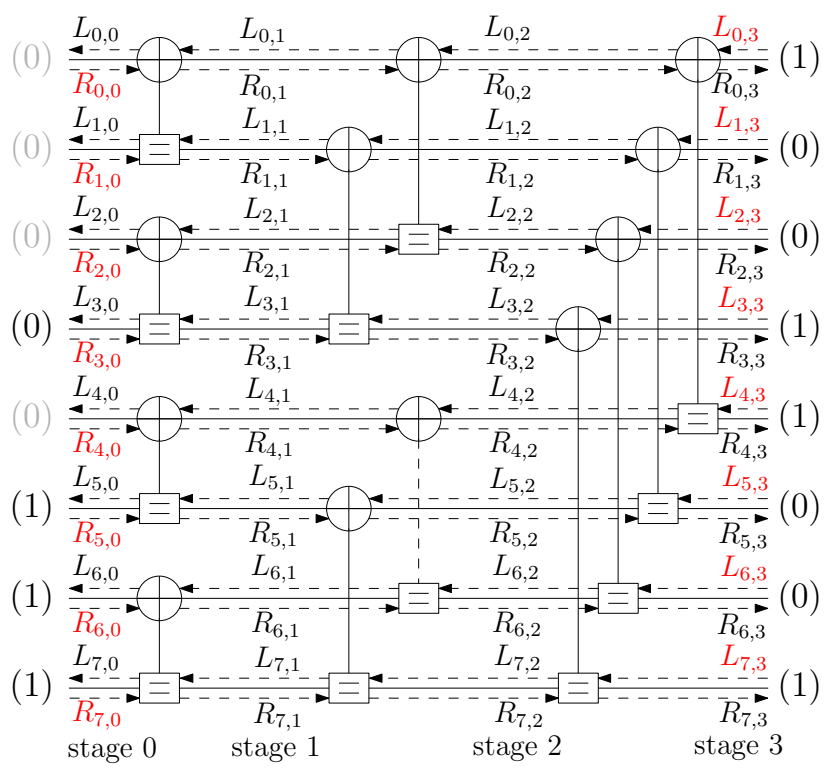

Fig. 1. The code graph of polar codes for the example of $N=8$.

- Finally, we compare our SSCS decoder with several stateof-the-art soft-output polar decoders in terms of its BER, block error ratio (BLER) and complexity. Our simulation results show that the proposed SSCS decoder outperforms the state-of-the-art soft-output polar decoders, including the BP [13], SCAN [14] and soft list [19] polar decoders.

The rest of this compact Letter is structured as follows. Section II describes the proposed SSCS decoder, whereas Section III details our polar-coded large-scale MIMO system employing the SSCS decoder. Then the system's performance quantified in terms of its BER vs. complexity is characterised in Section IV. Finally, our main conclusions are summarized in Section V.

\section{Soft-output Polar Decoding}

In Section II-A, we first briefly review polar encoding, followed by our proposed SSCS decoder in Section II-B.

\section{A. Polar encoding}

Firstly, an $A$-bit information sequence together with $(N-$ $A)$ frozen bits is polar-encoded into a polar-encoded sequence comprising of $N=2^{K}(N \geq A)$ bits. Polar encoding is carried out by the modulo- 2 matrix multiplication $\mathbf{u}_{K}=\mathbf{u}_{0} \mathbf{F}_{2}^{\otimes K}$ [1], where $\mathbf{F}_{2}^{\otimes K}$ is the generator matrix, and the superscript $\otimes K$ indicates the $K$-th Kronecker power of the matrix $\mathbf{F}_{2}$, which can be expressed as $\mathbf{F}_{2}=\left[\begin{array}{ll}1 & 0 \\ 1 & 1\end{array}\right]$.

Fig. 1 exemplifies this encoding operation, where the $A=$ $4, N=8$ uncoded block is $\mathbf{u}_{0}=[00000111]$, where $u_{3,0}, u_{5,0}$, $u_{6,0}$, and $u_{7,0}$ are the information bits, which corresponds to the 4 most polarized bit channels, and the remaining $u_{3,0}, u_{5,0}$, $u_{6,0}$, and $u_{7,0}$ are the frozen bits with zero values. While $\mathbf{u}_{0}=$ [00000111] comprises the left input of the code graph of Fig. 1, the polar encoded frame $\mathbf{u}_{3}=[10011001]$ is generated at the right-hand side of the code graph, after $K=3$ stages of XOR operations.

Then different rate matching operations, including repetition, shortening and puncturing, may be applied to the encoded frame, as in the 3GPP New Radio (NR) polar code [11], resulting in a $[A, G]$ polar code having a code-rate of $R=A / G$.

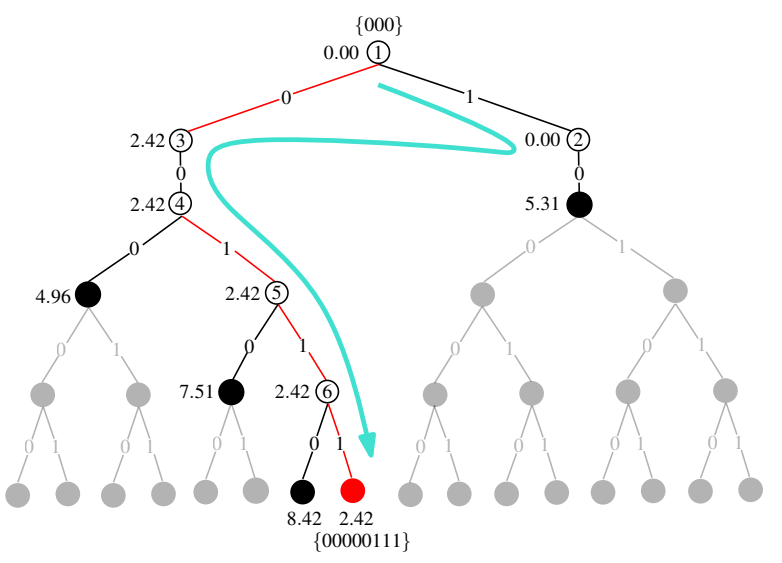

Fig. 2. An example of the code tree of SCS decoder having a stack-size of $S=2$.

\section{B. Soft successive cancellation stack (SSCS) polar decoding algorithms}

The operations of the SSCS polar decoder are summarized in Algorithm 1. The SSCS decoding commences with the SCS scheduling operations of [11]. Specifically, given the input LLRs $\mathbf{L}_{K}$, a path metric (PM) is first calculated and stored in a stack having a size $S$ for quantifying the likelihoods of a certain decoding candidate sequence, which is expressed as [11]

$$
\begin{aligned}
P M_{n} & =P M_{n-1}+\ln \left(1+\exp \left[-\left(1-2 \tilde{u}_{n, 0} L_{n, 0}\right)\right]\right) \\
& \approx\left\{\begin{array}{cc}
P M_{n-1}, & \text { if } \tilde{u}_{n, 0}=\frac{1}{2}\left[1-\operatorname{sign}\left(L_{n, 0}\right)\right] ; \\
P M_{n-1}+\left|L_{n, 0}\right|, & \text { otherwise, }
\end{array}\right.
\end{aligned}
$$

where $P M_{0}=0$ and $L_{n, 0}$ is the LLR of the $n$-th ( $n=$ $1,2, \cdots, N)$ bit at stage 0 , which is defined as $L_{n, 0}=$ $\ln \frac{\operatorname{Pr}\left(\tilde{u}_{n, 0}=0\right)}{\operatorname{Pr}\left(\tilde{u}_{n, 0}=1\right)}$. The calculation of PMs is demonstrated in Lines 5-13 of Algorithm 1, where pmin, pmax and sndpmin represent the position index of the decoding candidate having lowest, highest and second lowest PM value in the stack, respectively, while $P L[$ pmin $]$ counts the sequence length of the decoding candidate in the pmin-th position of the stack.

An example of the SCS polar decoder having a stack-size of $S=2$ dealing with PMs for decoding the polar code $\mathbf{u}_{3}=$ [10011001] is shown in Fig. 2. We can see that the depth-first search of the SCS decoder proceeds with the top element in the stacks, which is the candidate sequence having the lowest PM, giving $s \leq S$ variable-length candidate sequences. In order to avoid visiting a single bit too many times, a counter $P V[n]$ is introduced for counting the number of visiting times of the $n$ th bit [19]. When $P V[n]$ reaches a pre-set value, which is $S / 2$ in this letter, all the candidates that have a shorter length than $n$ in the stack will be popped out from the stack for releasing extra memory, as shown in Lines 26-27 of the Algorithm 1. The search process is demonstrated in Lines 8-29 of Algorithm 1 , where the sorting operation follows [10].

As soon as we obtained the $N$-length decoding candidate sequence having the best PM, the hard decisions of the corresponding bits are carried out based on the bit LLRs. Then the CRC is performed on the decoded bit sequence. If CRC is passed, the decoding process terminated and the corresponding candidate sequence is treated as the decoded bit sequence. On the contrary, if all $S$ candidate sequences fail to pass the CRC, we then generate the soft information for subsequent IDD, as will be detailed in Section III.

The generation of soft outputs of the SSCS decoder is summarized in Algorithm 2. Specifically, the hard decisions $\hat{\mathbf{u}}_{0}$ 

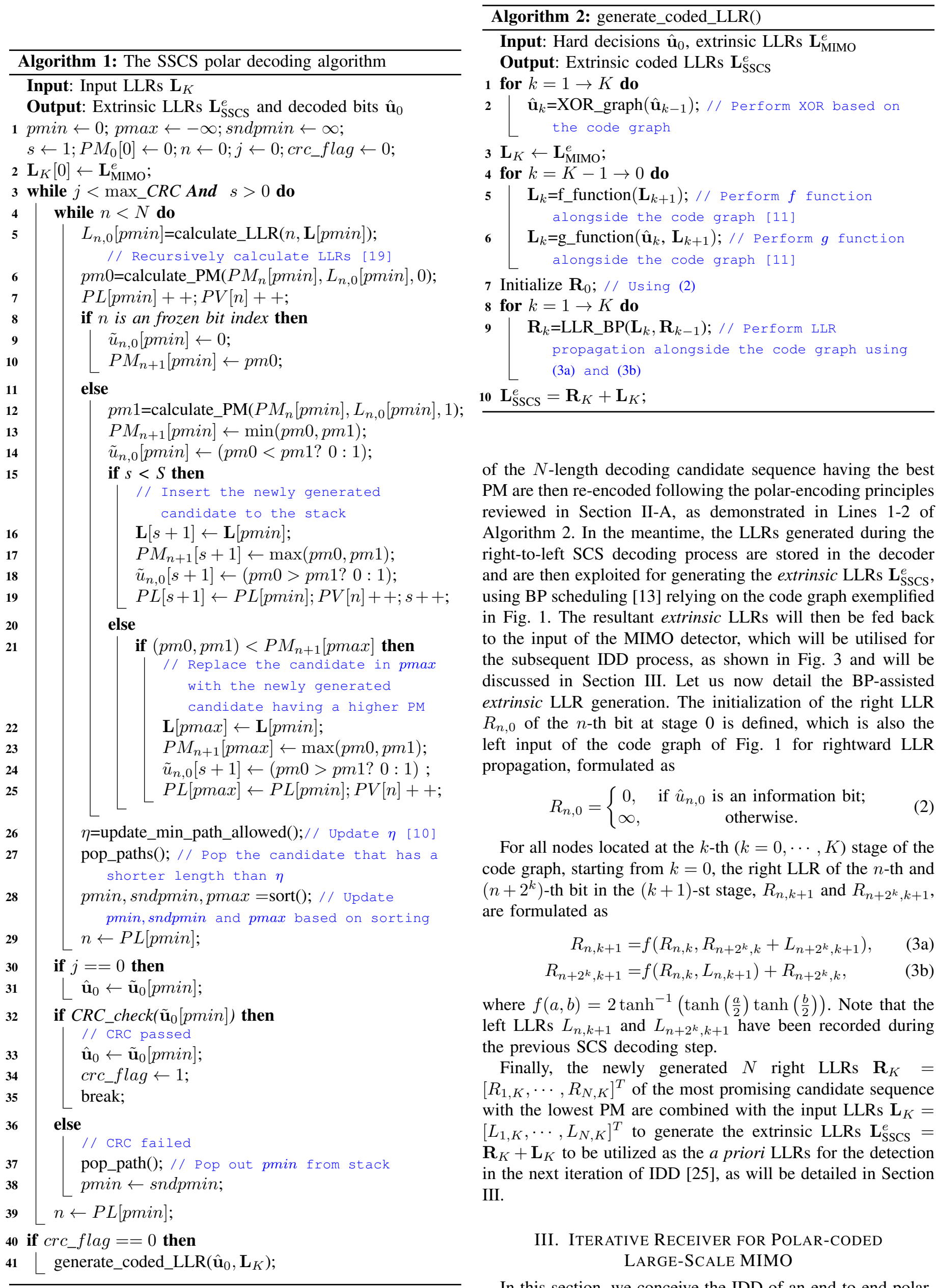

of the $N$-length decoding candidate sequence having the best PM are then re-encoded following the polar-encoding principles reviewed in Section II-A, as demonstrated in Lines 1-2 of Algorithm 2. In the meantime, the LLRs generated during the right-to-left SCS decoding process are stored in the decoder and are then exploited for generating the extrinsic LLRs $\mathbf{L}_{\text {SSCS }}^{e}$, using BP scheduling [13] relying on the code graph exemplified in Fig. 1. The resultant extrinsic LLRs will then be fed back to the input of the MIMO detector, which will be utilised for the subsequent IDD process, as shown in Fig. 3 and will be discussed in Section III. Let us now detail the BP-assisted extrinsic LLR generation. The initialization of the right LLR $R_{n, 0}$ of the $n$-th bit at stage 0 is defined, which is also the left input of the code graph of Fig. 1 for rightward LLR propagation, formulated as

$$
R_{n, 0}=\left\{\begin{array}{cc}
0, & \text { if } \hat{u}_{n, 0} \text { is an information bit; } \\
\infty, & \text { otherwise. }
\end{array}\right.
$$

For all nodes located at the $k$-th $(k=0, \cdots, K)$ stage of the code graph, starting from $k=0$, the right LLR of the $n$-th and $\left(n+2^{k}\right)$-th bit in the $(k+1)$-st stage, $R_{n, k+1}$ and $R_{n+2^{k}, k+1}$, are formulated as

$$
\begin{aligned}
R_{n, k+1} & =f\left(R_{n, k}, R_{n+2^{k}, k}+L_{n+2^{k}, k+1}\right), \\
R_{n+2^{k}, k+1} & =f\left(R_{n, k}, L_{n, k+1}\right)+R_{n+2^{k}, k},
\end{aligned}
$$

where $f(a, b)=2 \tanh ^{-1}\left(\tanh \left(\frac{a}{2}\right) \tanh \left(\frac{b}{2}\right)\right)$. Note that the left LLRs $L_{n, k+1}$ and $L_{n+2^{k}, k+1}$ have been recorded during the previous SCS decoding step.

Finally, the newly generated $N$ right LLRs $\mathbf{R}_{K}=$ $\left[R_{1, K}, \cdots, R_{N, K}\right]^{T}$ of the most promising candidate sequence with the lowest PM are combined with the input LLRs $\mathbf{L}_{K}=$ $\left[L_{1, K}, \cdots, L_{N, K}\right]^{T}$ to generate the extrinsic LLRs $\mathbf{L}_{\text {SSCS }}^{e}=$ $\mathbf{R}_{K}+\mathbf{L}_{K}$ to be utilized as the a priori LLRs for the detection in the next iteration of IDD [25], as will be detailed in Section III.

\section{ITERATIVE RECEIVER FOR POLAR-CODED LARGE-SCALE MIMO}

In this section, we conceive the IDD of an end-to-end polarcoded large-scale MIMO V-BLAST system, where the transmitter is equipped with $N_{t}$ transmit antennas (TAs) whereas 


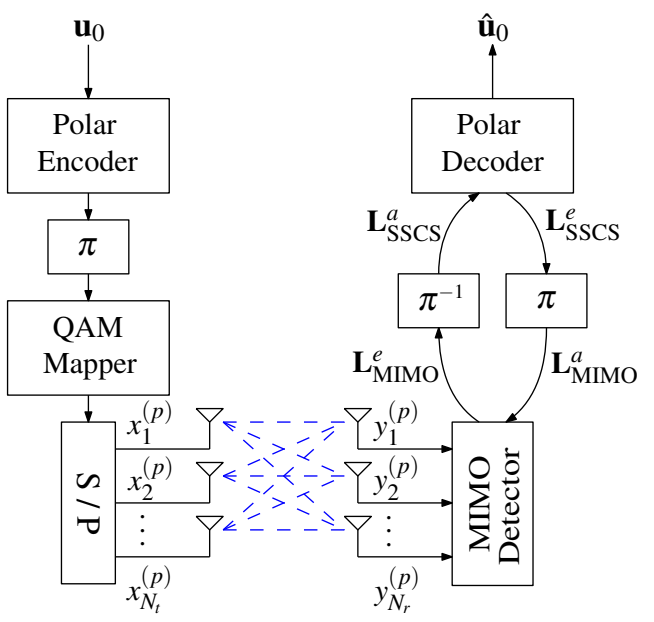

Fig. 3. The transceiver structure of the polar-coded MIMO system.

the receiver employs $N_{r}$ receive antennas (RAs), as shown in Fig. 3. Specifically, after the polar-encoding process discussed in Section II-A, the encoded bits of sequence $\mathbf{u}_{K}$ are interleaved and mapped to $Q$ symbols by $M$-ary quadrature amplitude modulation (QAM) having a constellation $\mathcal{M}=$ $\left\{s_{1}, \cdots, s_{M}\right\}$, where we have $Q=G / \log _{2} M$. After the serial-to-parallel (S/P) converter of Fig. 3, the $Q$ QAM symbols $\mathbf{X}=\left[x_{1}, \cdots, x_{Q}\right]^{T}$ are transmitted by $N_{t}$ TAs over the $Q / N_{t}$ symbol durations.

Given the channel impulse response $(\mathrm{CIR}) h_{r, t}^{(p)}$ between the $t$-th TA and the $r$-th RA, the observations at the receiver in the $p$-th $\left(p=1,2, \cdots, Q / N_{t}\right)$ symbol duration are expressed as

$$
\mathbf{y}^{(p)}=\mathbf{H}^{(p)} \mathbf{x}^{(p)}+\mathbf{n}^{(p)}, 1 \leq p \leq Q / N_{t},
$$

where $\mathbf{y}^{(p)} \in \mathbb{C}^{N_{r} \times 1}$ represnts the received observations of the $N_{r}$ RAs in the $p$-th symbol duration, $\mathbf{H}^{(p)} \in \mathbb{C}^{N_{r} \times N_{t}}$ is the channel matrix, $\mathbf{x}^{(p)}=\left[x_{(p-1) N_{t}+1}, \cdots, x_{p N_{t}}\right]^{T}$ is the transmitted symbol vector and $\mathbf{n}^{(p)} \in \mathbb{C}^{N_{r} \times 1}$ is the Additive White Gaussian Noise (AWGN) vector having a zero mean and a covariance matrix of $\mathbf{I}_{\sigma^{2}}$, where $\sigma^{2}$ is the signal-to-noise ratio (SNR).

In the $p$-th symbol duration, the received signal $\mathbf{y}^{(p)}$ is first input to the MIMO detector, where low-complexity minimum mean square error-interference cancellation (MMSE-IC) detection [26] is performed to give the detected signal $\hat{\mathbf{x}}^{(p)}$. This is in contrast to the example of a $(2 \times 2)$ polar-coded MIMO system of [19], where the maximum-likelihood (ML) MIMO detection could be implemented with affordable complexity ${ }^{1}$. In this Letter, we assume that the channel experiences uncorrelated Rayleigh fading, hence omitting the superscript $(p)$ in the following discussion for simplicity. Specifically, the output $Z_{t}$ of the MIMO detector detecting the QAM symbol transmitted by $t$-th TA can be expressed as

$$
Z_{t}=\mathbf{W}_{t}^{H} \mathbf{y}
$$

where $\mathbf{W}_{t}$ is the MMSE weight matrix, which is defined as [26] $\mathbf{W}_{t}=\left\{\mathbf{H}_{t} \mathbf{H}_{t}^{H}+\mathbf{H}_{\bar{t}}\left[\mathbf{R}_{x x}^{\bar{t}}-\operatorname{diag}\left(\tilde{\mathbf{x}}_{\bar{t}}^{H} \tilde{\mathbf{x}}_{\bar{t}}\right)\right] \mathbf{H}_{\bar{t}}^{H}+\sigma^{2} \mathbf{I}_{N_{r}}\right\}^{-1} \mathbf{H}_{t}$

In (6), $\mathbf{H}_{\bar{t}}$ is comprised of $\mathbf{H}_{t}$, excluding the $t$-th column and $\mathbf{x}_{\bar{t}}$ is comprised of $\mathbf{x}$ excluding $x_{t}$, respectively, so that $\mathbf{H}_{\bar{t}}^{H} \mathbf{x}_{\bar{t}}$ represents the inter-antenna interference, which may be

\footnotetext{
${ }^{1}$ Note that various low-complexity MIMO detectors such as BP [27], approximate message passing (AMP) [28] and K-best sphere decoding [29] are also perfectly compatible with our polar-coded MIMO system.
}

cancelled when using our MMSE-IC scheme. Additionally, $\tilde{x}_{t}$ is the estimated symbol based on the a priori LLRs, expressed as

$$
\begin{aligned}
\tilde{x}_{t} & =\sum_{m=1}^{M} s_{m} \cdot \operatorname{Pr}\left(\tilde{x}_{t}=s_{m}\right), \\
& =\sum_{m=1}^{M} s_{m} \cdot \frac{\exp \left[\sum_{d=1}^{\log _{2} M}\left(1-b_{d, t}^{\left(s_{m}\right)}\right) L_{\mathrm{MIMO}}^{a}\left(b_{d, t}\right)\right]}{\prod_{d=1}^{\log _{2} M}\left\{1+\exp \left[L_{\mathrm{MIMO}}^{a}\left(b_{d, t}\right)\right]\right\}},
\end{aligned}
$$

where $s_{m} \in \mathcal{M}, b_{d, t}^{\left(s_{m}\right)}$ represents the bit mapping corresponding to the symbol $s_{m}, L_{\mathrm{MIMO}}^{a}\left(b_{d, t}\right)$ denotes the LLR of the $d$-th bit in the QAM symbol $x_{t}$ and $\mathbf{R}_{x x}=\operatorname{diag}\left(\tilde{x}_{1}, \cdots, \tilde{x}_{N_{t}}\right)$.

Then the extrinsic bit LLR $L_{\mathrm{MIMO}}^{e}\left(b_{d, t}\right)$ of the $d$-th bit of the QAM symbol $x_{t}$ is calculated for forwarding to the SSCS polar decoder for IDD, which can be expressed as

$$
L_{\mathrm{MIMO}}^{e}\left(b_{d, t}\right)=L_{\mathrm{MIMO}}^{\text {post }}\left(b_{d, t}\right)-L_{\mathrm{MIMO}}^{a}\left(b_{d, t}\right),
$$

where $L_{\mathrm{MIMO}}^{\text {post }}\left(b_{d, t}\right)$ is expressed as

$$
L_{\mathrm{MIMO}}^{\text {post }}\left(b_{d, t}\right)=\ln \frac{\sum_{s_{m} \in \mathcal{M}, b_{d, t}=0} p_{m}}{\sum_{s_{m} \in \mathcal{M}, b_{d, t}=1} p_{m}},
$$

where $p_{m}$ is formulated as [26]

$$
p_{m}=-\frac{\left|\bar{h}_{r, t}\left(\tilde{Z}_{t}-s_{m}\right)\right|^{2}}{\left\|\mathbf{W}_{t}\right\|^{2} \cdot \sigma^{2}}+\sum_{d=1}^{\log _{2} M}\left(1-b_{d, t}^{\left(s_{m}\right)}\right) L_{\mathrm{MIMO}}^{a}\left(b_{d, t}\right),
$$

in conjunction with $\bar{h}_{r, t}=\mathbf{W}_{t}^{H} \mathbf{H}_{t}, \tilde{Z}_{t}=\bar{Z}_{t} \cdot \bar{h}_{r, t}^{*} /\left|\bar{h}_{r, t}\right|^{2}$, and $\bar{Z}_{t}$ is the output after the IC, which can be expressed as

$$
\bar{Z}_{t}=Z_{t}-\mathbf{W}_{t}^{H} \mathbf{H}_{\bar{t}} \tilde{\mathbf{x}}_{\bar{t}} .
$$

Following parallel-to-serial (P/S) conversion and deinterleaving, the SSCS polar decoding operation of Section II-B is carried out by processing the input LLRs $\mathbf{L}_{\mathrm{SSCS}}^{a}=$ $\pi\left(\mathbf{L}_{\text {MIMO }}^{e}\right)$ gleaned from the MIMO detector, where $\mathbf{L}_{\text {MIMO }}^{e}=$ $\left[L_{\text {MIMO }}^{e}\left(b_{1,1}^{(1)}\right), \cdots, L_{\text {MIMO }}^{e}\left(b_{d, t}^{(p)}\right), \cdots, L_{\text {MIMO }}^{e}\left(b_{\log _{2} M, N_{t}}^{\left(Q / N_{t}\right)}\right)\right]^{T}$ and $L_{\text {MIMO }}^{e}\left(b_{d, t}^{(p)}\right)$ is given in (8). As discussed in Section II-B, if the decoding candidates fail to pass the CRC, $\mathbf{L}_{\text {SSCS }}^{e}$ will be input to the MIMO detector as a priori LLRs in the following iteration, facilitating the IDD.

\section{PERformance of SSCS DECODER IN POLAR-CODED MIMO}

In this section, we characterize the proposed SSCS decoder aided polar-coded large-scale MIMO V-BLAST system for communication over uncorrelated Rayleigh fading channels in terms of BER and BLER in Section IV-A, as well as complexity in Section IV-B. The simulation parameters are summarised in Table II.

TABLE II

SIMULATION PARAMETERS OF SECTION IV

\begin{tabular}{l|r}
\hline Parameters & Values \\
\hline Polar code $[A, G]$ & {$[256,512]$} \\
\hline QAM order $M$ & 4 \\
\hline Number of TAs $N_{t}$ & 64 \\
\hline Number of RAs $N_{r}$ & 64 \\
\hline Number of outer iterations $I$ & 8 \\
\hline Number of CRC bits & 11 \\
\hline
\end{tabular}




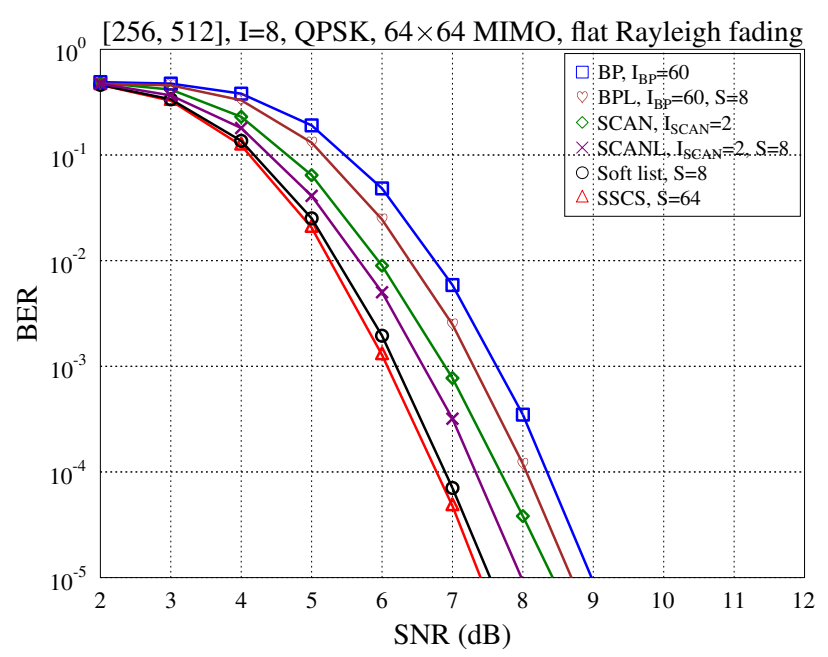

Fig. 4. BER comparison of the proposed SSCS polar decoder with stateof-the-art polar decoders, including BP [13], SCAN [14], BPL [16], SCANL [17] and soft list [19] polar decoders for the polar-coded $(64 \times 64) \mathrm{MIMO}$ system, where the $A=256, G=512$ polar codes are employed and $I=8$ outer iterations between the MIMO detector and polar decoder are used when communicating over uncorrelated Rayleigh fading channels.

\section{A. BER performance}

Firstly, we compare the BER performance of our SSCS polar decoder with that of several state-of-the-art soft-output decoder benchmarks. Explicitly, Fig. 4 contrasts the BER performance of the proposed SSCS polar decoder to the BP [13], SCAN [14], BPL [16], SCANL [17] and soft list [19] decoders using the parameters of Table II. Observe from Fig. 4 that for both frame lengths, our SSCS decoder achieves the best BER performance among these soft-output polar decoder candidates. More specifically, since BPL and SCANL perform independent $\mathrm{BP}$ and SCAN decoding algorithms on a list of permuted polar codes, respectively, they achieves superior BER performance over the BP and SCAN decoding. Additionally, the proposed SSCS polar decoder having a stack size of $S=64$ is capable of slightly outperforming the soft list decoder of [19] using a list size of $S=8$. Even though a higher stack size is required for our SSCS polar decoder than that of soft list decoder, this performance improvement is achieved without imposing extra complexity in the high-SNR regions, as it will be demonstrated in the next section.

Furthermore, the influence of the stack size $S$ on the BER and BLER performance is characterized in Figs. 5 and 6, respectively, where we have $S=16,32,64$ and 128 in our SSCS polar decoder. We can see that the increase of $S$ gradually leads to slightly superior BER performance compared to the bestperforming soft list polar decoder having $S=8$ [19]. However, if we increase the list size of the soft list polar decoder, the BER performance will also be improved, as discussed in [19]. Furthermore, compared with the non-iterative hard-output SCS decoder, a $1.5 \mathrm{~dB}$ SNR gain can be observed from Fig. 5 at a BER level of $10^{-5}$, when the $S=64$ SSCS polar decoder is employed. Similar conclusions can be observed for the BLER. By comparing Figs. 5 and 6, we can see that when SSCS is employed, only a slightly higher SNR is required to achieve a BLER of $10^{-5}$ than that for attaining the $10^{-5}$ BER. This is because when CRC is applied, the SSCS decoder carries out the soft decisions only when the entire block passes CRC.

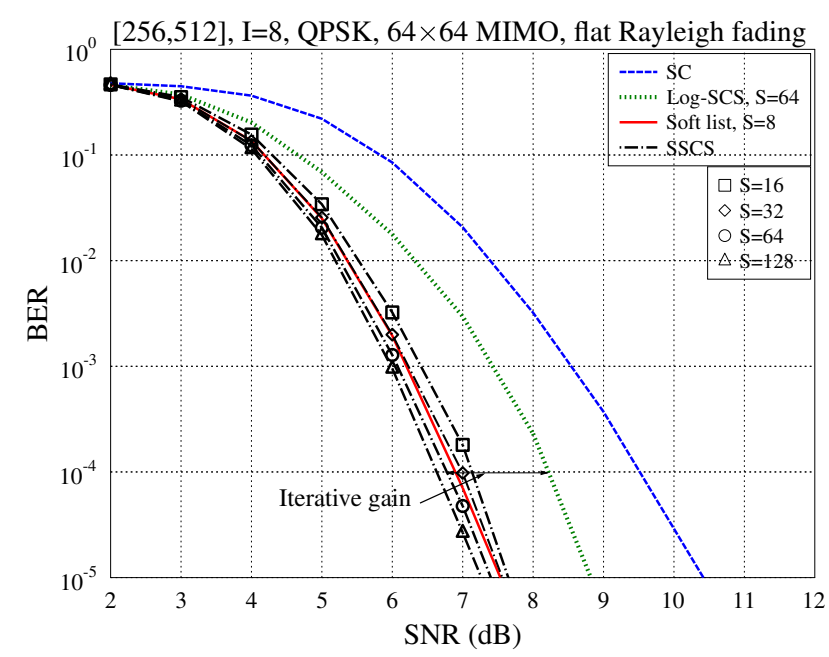

Fig. 5. BER performance of the proposed SSCS polar decoder with different stack sizes of $S=16,32,64$ and 128 for polar-coded $(64 \times 64)$ MIMO system using $I=8$ outer iterations, where $A=256, G=512$ polar code is employed for communication over uncorrelated Rayleigh fading channels.

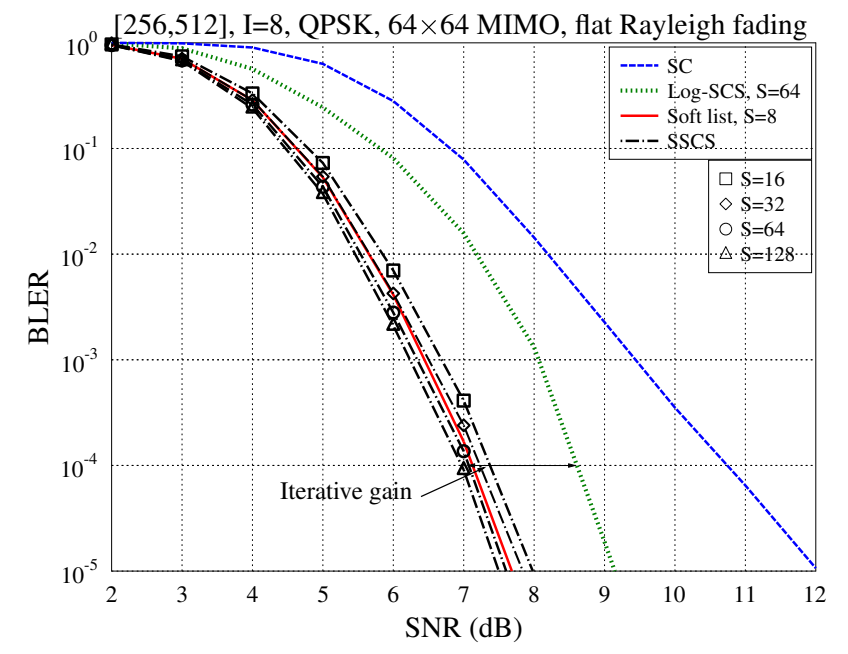

Fig. 6. BLER performance of the proposed SSCS polar decoder with $S=16,32,64$ and 128 for polar-coded $(64 \times 64)$ MIMO system using $I=8$ outer iterations, where $A=256, G=512$ polar code is employed for communication over uncorrelated Rayleigh fading channels.

\section{B. Complexity}

In this compact Letter, we quantify the decoding complexity by counting the number of Add, Compare and Select (ACS) operations required for both the benchmark and the proposed polar decoders. Fig. 7 characterizes the complexity of the BP, SCAN, soft list and the proposed SSCS polar decoder required for completing a single outer iteration in the context of our polar-coded MIMO system using $N_{t}=N_{r}=64$, where again, the $A=256, G=512$ polar code is employed for communicating over uncorrelated Rayleigh fading channels. Observe from Fig. 7 that the original BP decoder suffers from a high complexity due to the high number of iterations required for achieving the BLER of the SCAN and of the soft list polar decoders. Note that by applying early stopping techniques [15], the decoding complexity of the BP decoder can be further reduced by about $25 \%$ in the high-SNR region. However, this is still far higher than that of our proposed SSCS decoder. By contrast, the SCAN decoder imposes a lower decoding complexity than our SSCS decoder in the low-SNR region, but 


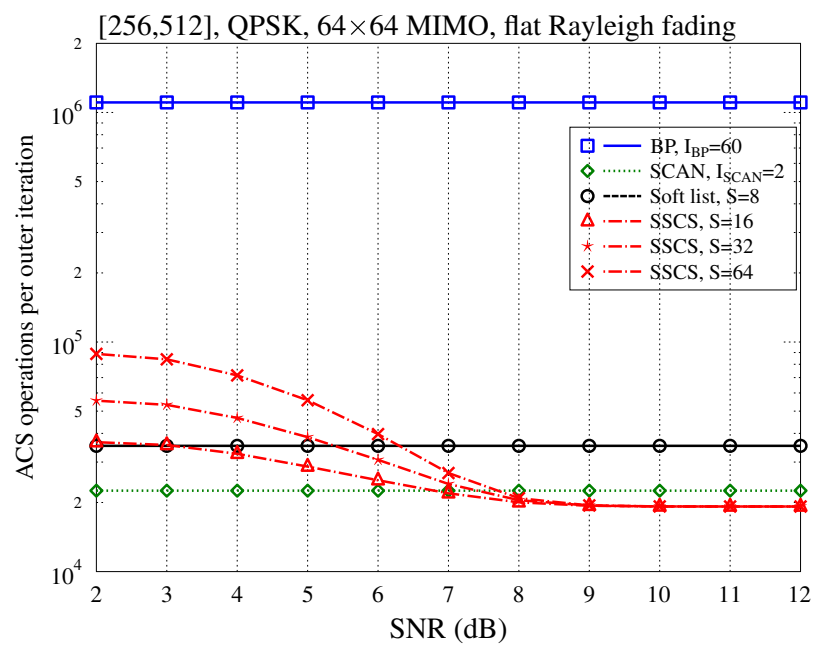

Fig. 7. Decoding complexity of the SCS, BP, SCAN, soft list and the proposed SSCS polar required for completing a single outer iteration in polar-coded $(64 \times 64)$ MIMO system, where $A=256, G=512$ polar code is employed for communicating over uncorrelated Rayleigh fading channels.

it suffers from about $1 \mathrm{~dB}$ performance loss at a BER of $10^{-5}$, as characterized in Section IV-A. Note that the decoding latency is also related to the decoding complexity. However, since the decoding of $S$ candidates can be performed simultaneously during the right-to-left SCS decoding, achieving a rather low latency is possible, as demonstrated in [12]. In the next stage of rightward BP scheduling, decoding of the entire block having $N$ bits can be performed in parallel, as discussed in [15].

\section{CONCLUSION}

A novel SSCS polar decoder has been proposed in this Transaction Brief, which achieves superior BER performance over the SCS decoder and the state-of-the-art soft-output decoders. Furthermore, our SSCS decoder was intrinsically amalgamated with a polar-coded large-scale MIMO system and an IDD was conceived, operating on the basis of exchanging soft-LLRs. Our simulation results show that the proposed SSCS decoder is capable of attaining a better BER performance than the best-performing state-of-the-art soft list polar decoder, despite imposing a $50 \%$ lower complexity at moderate to high SNRs. Compared to the non-iterative hard-output SCS decoder, our SSCS attained about $1.5 \mathrm{~dB}$ SNR gain at a BER level of $10^{-5}$ in the polar-coded $(64 \times 64)$ MIMO system considered.

\section{REFERENCES}

[1] E. Arıkan, "Channel polarization: A method for constructing capacityachieving codes for symmetric binary-input memoryless channels," IEEE Transactions on Information Theory, vol. 55, no. 7, pp. 3051-3073, 2009.

[2] Z. B. K. Egilmez, L. Xiang, R. G. Maunder, and L. Hanzo, "The development, operation and performance of the $5 \mathrm{G}$ polar codes," IEEE Communications Surveys \& Tutorials, vol. 22, no. 1, pp. 96-122, 2019.

[3] Z. Babar, Z. B. K. Egilmez, L. Xiang, D. Chandra, R. G. Maunder, S. X. $\mathrm{Ng}$, and L. Hanzo, "Polar codes and their quantum-domain counterparts," IEEE Communications Surveys \& Tutorials, vol. 22, no. 1, pp. 123-155, 2019.

[4] M. Shirvanimoghaddam, M. S. Mohammadi, R. Abbas, A. Minja, C. Yue, B. Matuz, G. Han, Z. Lin, W. Liu, Y. Li, et al., "Short block-length codes for ultra-reliable low latency communications," IEEE Communications Magazine, vol. 57, no. 2, pp. 130-137, 2018.

[5] I. Tal and A. Vardy, "List decoding of polar codes," IEEE Transactions on Information Theory, vol. 61, no. 5, pp. 2213-2226, 2015.

[6] K. Niu and K. Chen, "Stack decoding of polar codes," Electronics letters, vol. 48, no. 12, pp. 695-697, 2012.
[7] H. Zhou, W. Song, W. J. Gross, Z. Zhang, X. You, and C. Zhang, "An efficient software stack sphere decoder for polar codes," IEEE Transactions on Vehicular Technology, vol. 69, no. 2, pp. 1257-1266, 2020.

[8] H. Zhou, W. J. Gross, Z. Zhang, X. You, and C. Zhang, "Efficient sphere polar decoding via synchronous determination," IEEE Transactions on Vehicular Technology, 2020.

[9] A. Balatsoukas-Stimming, M. B. Parizi, and A. Burg, "LLR-based successive cancellation list decoding of polar codes," IEEE Transactions on Signal Processing, vol. 63, no. 19, pp. 5165-5179, 2015.

[10] H. Aurora, C. Condo, and W. J. Gross, "Low-complexity software stack decoding of polar codes," in Circuits and Systems (ISCAS), 2018 IEEE International Symposium on, pp. 1-5, IEEE, 2018.

[11] L. Xiang, Z. B. K. Egilmez, R. G. Maunder, and L. Hanzo, "CRC-aided logarithmic stack decoding of polar codes for ultra reliable low latency communication in 3GPP new radio," IEEE Access, vol. 7, pp. 2855928573, 2019

[12] L. Xiang, S. Zhong, R. G. Maunder, and L. Hanzo, "Reduced-complexity low-latency logarithmic successive cancellation stack polar decoding for $5 \mathrm{~g}$ new radio and its software implementation," IEEE Transactions on Vehicular Technology, vol. 69, no. 11, pp. 12449-12458, 2020.

[13] E. Arrkan, "Polar codes: A pipelined implementation," in Proc. 4th Int. Symp. on Broad. Commun. ISBC 2010, pp. 11-14, 2010.

[14] U. U. Fayyaz and J. R. Barry, "Low-complexity soft-output decoding of polar codes," IEEE Journal on Selected Areas in Communications, vol. 32, no. 5, pp. 958-966, 2014.

[15] B. Yuan and K. K. Parhi, "Early stopping criteria for energy-efficient low-latency belief-propagation polar code decoders," IEEE Transactions on Signal Processing, vol. 62, no. 24, pp. 6496-6506, 2014.

[16] A. Elkelesh, M. Ebada, S. Cammerer, and S. ten Brink, "Belief propagation list decoding of polar codes," IEEE Communications Letters, vol. 22, no. 8 , pp. $1536-1539,2018$.

[17] C. Pillet, C. Condo, and V. Bioglio, "SCAN list decoding of polar codes," in ICC 2020-2020 IEEE International Conference on Communications (ICC), pp. 1-6, IEEE, 2020.

[18] J. Jiao, K. Liang, B. Feng, Y. Wang, S. Wu, and Q. Zhang, "Joint channel estimation and decoding for polar coded SCMA system over fading channels," IEEE Transactions on Cognitive Communications and Networking, 2020.

[19] L. Xiang, Y. Liu, Z. B. K. Egilmez, R. G. Maunder, L.-L. Yang, and L. Hanzo, "Soft list decoding of polar codes," IEEE Transactions on Vehicular Technology, vol. 69, no. 11, pp. 13921-13926, 2020.

[20] B. Feng, J. Jiao, S. Wu, Y. Wang, and Q. Zhang, "Iterative and adjustable soft list decoding for polar codes," IEEE Transactions on Signal Processing, vol. 68, pp. 5559-5572, 2020.

[21] J. Dai, K. Niu, Z. Si, C. Dong, and J. Lin, "Polar-coded non-orthogonal multiple access," IEEE Transactions on Signal Processing, vol. 66, no. 5, pp. 1374-1389, 2017.

[22] E. Marshakov, G. Balitskiy, K. Andreev, and A. Frolov, "A polar code based unsourced random access for the Gaussian MAC," in 2019 IEEE 90th Vehicular Technology Conference (VTC2019-Fall), pp. 1-5, IEEE, 2019.

[23] M. Ebada, S. Cammerer, A. Elkelesh, M. Geiselhart, and S. t. Brink, "Iterative detection and decoding of finite-length polar codes in Gaussian multiple access channels," arXiv preprint arXiv:2012.01075, 2020.

[24] J. Dai, K. Niu, and J. Lin, "Polar-coded MIMO systems," IEEE Transactions on Vehicular Technology, vol. 67, no. 7, pp. 6170-6184, 2018.

[25] K. Han, J. Wang, W. J. Gross, and J. Hu, "Stochastic bit-wise iterative decoding of polar codes," IEEE Transactions on Signal Processing, vol. 67, no. 5, pp. 1138-1151, 2018.

[26] C. Xu, D. Liang, S. Sugiura, S. X. Ng, and L. Hanzo, "Reducedcomplexity approx-log-MAP and max-log-MAP soft PSK/QAM detection algorithms," IEEE Transactions on Communications, vol. 61, no. 4, pp. 1415-1425, 2013.

[27] P. Som, T. Datta, A. Chockalingam, and B. S. Rajan, "Improved largemimo detection based on damped belief propagation," in 2010 IEEE Information Theory Workshop on Information Theory (ITW 2010, Cairo), pp. 1-5, IEEE, 2010.

[28] S. Wu, L. Kuang, Z. Ni, J. Lu, D. Huang, and Q. Guo, "Low-complexity iterative detection for large-scale multiuser MIMO-OFDM systems using approximate message passing," IEEE Journal of Selected Topics in Signal Processing, vol. 8, no. 5, pp. 902-915, 2014.

[29] Z. Guo and P. Nilsson, "Algorithm and implementation of the k-best sphere decoding for mimo detection," IEEE Journal on selected areas in communications, vol. 24, no. 3, pp. 491-503, 2006. 